Conclusions There is a considerable demand for out-of-hours sleep clinics. This is predominantly from those in employment. Making up a large proportion of patients referred for suspected OSAH, over half of these patients preferred evening clinics. Provision of this clearly demanded service requires a change in current working practice. Not only would out-of-hours clinics be a move towards delivering the patient-centred NHS pledged in the recent Government White Paper ${ }^{1}$, but would deliver the increased clinic capacity required to meet rising demand.

${ }^{1}$ Equity and Excellence: Liberating the NHS. DoH. July 2010.

\section{P203 MANAGEMENT OF OBESITY IN RESPIRATORY CLINICS-ARE WE DOING ENOUGH?}

doi:10.1136/thx.2010.151068.4

S Mandal, D J Powrie. Southend University Hospital, Southend, UK

Introduction and Objectives Obesity is a growing problem and as Respiratory Physicians we are encountering more patients in clinics with co-morbidities associated with obesity. We wished to determine what services were available to patients with respiratory disease and obesity.

Methods We carried out a postal survey of respiratory departments in the UK.

Results 211 surveys were sent out and 100 were returned. 57 hospitals ran specialist sleep clinics, of those that did not have specialist clinics 41 saw obese patients in their general respiratory clinics. We asked about baseline measurements taken in clinic (BMI, waist circumference and collar size); 1 unit took no measurements; 23 took 1 measurement (most commonly BMI) and 60 took 2 measurements and 16 took all 3 measurements. 95 respondents gave their patients advice regarding the management of obesity. 1 hospital had a dietician available in clinic, 89 referred to a dietician; however, 10 had no access to a dietician at all. Physicians were asked about screening for co-morbidities. 81 units screened for hypertension, of these 47 (58\%) referred back to the GP for management, $12(15 \%)$ treated the hypertension themselves and 3 referred to specialists. 43 screened for diabetes, of these 18 (42\%) referred to the GP for management, 2 treated the diabetes themselves and 10 (23\%) referred to specialists. 16 screened for hypercholesterolaemia, 8 referred to the GP for management and $3(19 \%)$ treated the hypercholesterolaemia themselves. Physicians were asked what other forms of advice/services were available in clinic, Abstract P203 Table 1. Clinicians were asked to rate on a scale of $1-10$ about how happy they were with their current service, the average score was 4.55 . We then asked how they felt their service could be improved, most commonly clinicians felt a dietician should be present in clinic $(\mathrm{n}=22)$ and that the service/clinic should be in a multidisciplinary format $(n=16)$. Despite obesity and its co-morbidities being common, management in clinics is variable, as is access to services. An integrated pathway for the management of obesity may improve outcomes.

Abstract P203 Table 1 Modes of advice available in clinic

\begin{tabular}{lc}
\hline Advice given & Number \\
\hline Diet sheet & 24 \\
Refer to dietician & 80 \\
Exercise & 17 \\
Refer to obesity clinic & 29 \\
Refer to surgeons & 25 \\
No advice & 5 \\
\hline
\end{tabular}

\section{P204 TRAVEL WITH CPAP MACHINES: HOW FREQUENT AND WHAT ARE THE PROBLEMS?}

doi:10.1136/thx.2010.151068.5

S Faruqi, P Carveth-Johnson, A O C Johnson. Pontefract General Infirmary, Pontefract, UK

Introduction and Objectives Obstructive sleep apnoea (OSA) is common and most patients are relatively young. CPAP is the standard treatment. This questionnaire survey aimed to improve the understanding of the problems which patients on CPAP face during travel and while away from home.

Methods 150 patients on CPAP were randomly selected from our patient database and sent a postal questionnaire. They were asked the number of times they had travelled in the last 2 years and specific questions about the last three trips. These included mode of travel, duration of trip, change in CPAP use, problems with transport and use of CPAP. Patients were asked to specifically document whether they used CPAP during travel. All patients are offered a letter regarding their CPAP therapy to facilitate travel.

Results 101 questionnaires were returned (men=69, mean age $=60.5$ years). 58 ( $\mathrm{men}=50$, mean age 59.4 years) had travelled in the last 2 years on 211 occasions. Data were collected for 139 trips. In 13 trips the CPAP machine was not taken. Reasons included weight, space and check in concerns. 84 trips were within Europe and 7 to North America. 93 trips were by air. On 22 trips, respondents reported the "CPAP letter" was needed for check in. In 7 of the 93 trips by air, problems with checking in the CPAP machine were encountered. In all but 4 trips the CPAP machine was carried as hand luggage. In 33 trips problems with use of CPAP due to the short power cord was reported. CPAP was used less often abroad. CPAP was rarely used during travel. Individual statements highlighted problems with security and check-in.

Conclusions Patients with OSA travel abroad frequently and usually take their CPAP machine with them. In a significant number there are problems with check-in of the CPAP machine and we strongly recommend patients are provided with a "CPAP letter". Improved dissemination of information is needed to patients and airport staff regarding OSA and CPAP. We recommend patients carry extension cords and power adaptors. The reason for less use of CPAP abroad is not clear and needs further explanation.

\section{P205 OBSTRUCTIVE SLEEP APNOEA SCREENING-COST AND CLINICALLY EFFECTIVE IN A TIA CLINIC?}

doi:10.1136/thx.2010.151068.6

M Waller, A Hussey, A Morris, D Hargroves, B Prathibha. William Harvey Hospital, Ashford, UK

Several large epidemiological studies have shown a strong association between OSA and the development of cerebrovascular disease and there is growing evidence to support this finding. These include Transient Ischaemic attacks and stroke. There is increased morbidity and mortality rates in these patients. Conversely patients with Strokes are at increased risk of developing obstructive and central sleep apnoea. Early recognition and treatment of Obstructive sleep apnoea has been shown to prevent cerebrovascular events.

Aim To assess the prevalence of cerebrovascular events in a cohort of patients diagnosed with obstructive sleep apnoea in Ashford.

Methods 100 patients with a confirmed diagnosis of OSA were randomly selected to enter the study. Detailed data, about their demographics, co morbidities as well as sleep, were collected. 
Obstructive Sleep apnoea was classified as mild, moderate and severe based on AHI (mild $<15 / \mathrm{h}$, moderate $15-30 / \mathrm{h}$ and severe $>30 / \mathrm{h}$ ). TIA was defined as a neurological deficit which resolved completely within $24 \mathrm{~h}$ and was not associated with any changes on CT/MRI. Stroke was classified according to the territory involved and accompanied by CT/MRI changes. Cholesterol was checked in all the patients and associated Diabetes mellitus was noted as well.

Results In this cohort, more patients had severe sleep apnoea (62\%). There was an increased incidence of cerebrovascular morbidities in the severe group as compared with the mild and moderate groups. $20 \%$ of all patients had hypercholesterolaemia ( $45 \%$ in the severe group), $10 \%$ of all patients had Transient Ischaemic Attack (80\% in the severe group), $2 \%$ had Stroke (100\% in the severe group) and $16 \%$ had Diabetes mellitus.

Conclusions Our study showed significant cerebrovascular comorbidities in patients with obstructive sleep apnoea. There is evidence to suggest that effective treatment of the sleep apnoea improves cerebrovascular outcomes. The fact that most of our patients with cerebrovascular comorbidity had TIA rather than strokes suggest that there is a window of opportunity to prevent further events by effective treatment. This certainly reduces the physical, social and financial burden incurred by strokes. We suggest that all patients with TIA should be screened for Obstructive Sleep apnoea and treated appropriately.

\section{Abstract P205 Table 1}

\begin{tabular}{lccllll}
\hline Severity & $\begin{array}{l}\text { Male } \\
(\mathbf{7 7})\end{array}$ & $\begin{array}{l}\text { Female } \\
(\mathbf{2 3})\end{array}$ & $\begin{array}{l}\text { Hypercholesterolaemia } \\
\mathbf{( 2 0 )}\end{array}$ & $\begin{array}{l}\text { TIA } \\
\mathbf{( 1 0 )}\end{array}$ & $\begin{array}{l}\text { Stroke } \\
(\mathbf{2})\end{array}$ & $\begin{array}{l}\text { Diabetes } \\
(\mathbf{1 6})\end{array}$ \\
\hline Mild (8) & 6 & 2 & 4 & 0 & 0 & 3 \\
Moderate (30) & 20 & 10 & 7 & 2 & 0 & 8 \\
Severe (62) & 51 & 11 & 9 & 8 & 2 & 5 \\
\hline
\end{tabular}

\section{P206 COMPLIANCE WITH CPAP: SUBJECTIVE VERSUS OBJECTIVE METHODS OF ASSESSMENT AND REGIONAL VARIATIONS}

doi:10.1136/thx.2010.151068.7

${ }^{1} \mathrm{~A}$ Lal, ${ }^{1} \mathrm{~L}$ Dale, ${ }^{1} \mathrm{~T}$ Lucas, ${ }^{2} \mathrm{~B}$ Chakraborty, ${ }^{3} \mathrm{D}$ Banerjee, ${ }^{1} \mathrm{D}$ Brocklebank. ${ }^{1}$ Alexandra Hospital, Redditch, UK; ${ }^{2}$ Birmingham University, Birmingham, UK; ${ }^{3}$ Heartlands Hospital, Birmingham, UK

Background OSA is a significant public health problem which can be treated effectively with CPAP. Compliance of $4 \mathrm{~h} / \mathrm{night}$ is required to achieve clinical effectiveness. Sleep clinics regularly monitor CPAP compliance subjectively, for example, patient questionnaires, but such estimates may differ from actual compliance.

Aim The aim of the study was to measure errors in estimating compliance and to look for regional variations in degrees of error.

Methods This is a prospective, two-centre study, carried out in 2009-2010. Centre 1 is a Tertiary centre with a local population including large numbers of South Asian people and those with lower socio-economic status. Centre 2 is a District General Hospital with a predominantly Caucasian local population. Both centres have similar sleep clinic setups and routinely download CPAP machine hours. Subjective compliance was assessed by patient questionnaires and objective evidence of compliance was obtained from machine usage data simultaneously.

Results 107 patients were included from each centre. In centre 1, $80 \%$ patients over-estimated their compliance, the mean objective usage of CPAP was $5.0 \mathrm{~h} /$ night and the mean error in estimating compliance was $+2.2 \mathrm{~h} /$ night. In centre $2,52 \%$ of patients overestimated their compliance, the mean objective usage of CPAP was $5.67 \mathrm{~h} /$ night and the mean error in estimating compliance was
$+1.03 \mathrm{~h} /$ night. Patients in Centre 2 had significantly higher CPAP usage ( 5.67 vs. $5 \mathrm{~h} /$ night, $\mathrm{p}=0.02$ ) and a lower percentage of people over-estimating their compliance $(52 \%$ vs $80 \%, p<0.00001)$. Overall, there was still a significant error in estimating compliance, although this was lower in Centre 2 (1.03 vs $2.2 \mathrm{~h} /$ night, $\mathrm{p}<0.00001$ ).

Discussion This study highlights the fact that patients tend to be significantly inaccurate about their compliance. Reasons for this are uncertain but may include aiming to please the health professional, poor cognitive insight into their usage and fear of relinquishing their machine. Electronic assessment of CPAP usage data should therefore be routine in all sleep clinics. Furthermore, there seem to be regional variations both in usage and in degrees of error. This may be attributed to differences in education levels and socio-economic status. Ethnicity may also contribute because of different cultural beliefs and lifestyles.

\section{Abstract P206 Table 1}

\begin{tabular}{lccl}
\hline & Centre 1 & Centre 2 & p-Value \\
\hline Mean usage (h/night) & 5.0 & 5.67 & $\mathrm{p}=0.02$ \\
Mean subjective error (h/night) & 2.2 & 1.03 & $\mathrm{p}<0.00001$ \\
Percentage of over-estimators (\%) & 80 & 52 & $\mathrm{p}=0.00002$ \\
\hline
\end{tabular}

\section{P207 OBSTRUCTIVE SLEEP APNOEA IN PATIENTS UNDERGOING BARIATRIC SURGERY - A LONDON TEACHING HOSPITAL EXPERIENCE}

doi:10.1136/thx.2010.151068.8

C J Jolley, S Zimmerman, S Shah, N Davison, S Singh. Chelsea \& Westminster Hospital NHS Foundation Trust, London, UK

Introduction and Objectives Obstructive sleep apnoea (OSA) is common in patients scheduled for bariatric surgery and increases the likelihood of peri-operative adverse events. We aimed to describe the prevalence of OSA and risk profile of patients referred for pre-bariatric assessment locally.

Methods A retrospective observational study of patients referred to the sleep clinic for assessment before bariatric surgery between June 2008 and February 2010. Clinical and anthropometric data were collected from the hospital notes and sleep studies. Patient-reported STOP BANG model scores were recorded or derived retrospectively from clinical data. Non-parametric statistics were used due to nonnormally distributed data.

Results 140/164 patients referred were seen in clinic. Referral rates increased from 2/month to $15 /$ month in February 2010. The median (range) age was 46.5 (18-68)years, 71\% female, weight 135.5 $(87.4-180 \mathrm{~kg})$, BMI $\left.48.4(35.3-84.5) \mathrm{kg} / \mathrm{m}^{2}\right)$, and median ESS was $11(0-24)$. STOP BANG scores were reported or could be calculated in 84 patients, in whom the median score was $5 / 8(2-8)$. When incomplete STOP BANG scores were included, 124/130 scored $>2$. $53 \%$ were non-smokers, $15 \%$ current smokers and were $37 \%$ exsmokers. Comorbidities included: diabetes/IGT 70\%, hypertension 50\%, hypercholesterolaemia 39\%, ACS/heart failure 9\%, CVA 2\%, COPD $3 \%$, asthma 19\%, and hypothyroidism 19\%. Sleep studies were requested for 129 patients, completed in 116 patients. 114 were technically adequate for AHI and 106 for pulse oximetry. The median total AHI was $10.5(0-111.2) / \mathrm{h}$, ODI $20.8(0.2-145.2) / \mathrm{h}$, and mean $\mathrm{SpO}_{2} 93.4$ (78.3-98.6)\%. 27\% had AHI $\geq 5 / \mathrm{h}, 12 \% \geq 15 / \mathrm{h}$ and $25 \% \geq 30 / \mathrm{h}$. Correlations between sleep study outcomes and clinical data are shown in Abstract P207 Table 1. Using STOP BANG $>2$ to screen for AHI $\geq 5$ had a sensitivity and specificity of $99.0 \%$ (95\% CI 92.4 to $100 \%$ ) and $5.6 \%$ (0.7-18.7\%), respectively. 